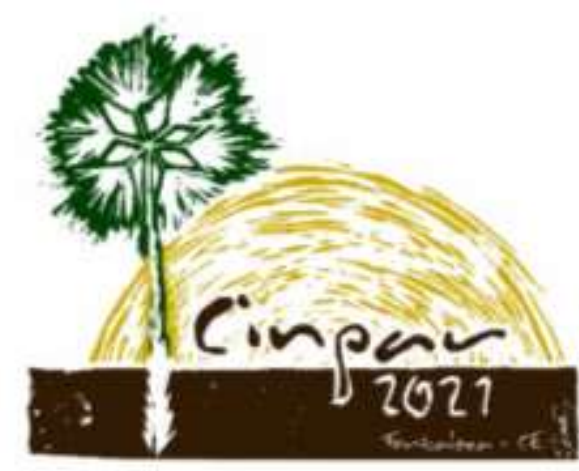

XVII Congresso Internacional sobre Patologia e Reabilitação das Construçōes

XVII Congreso Internacional sobre Patologia y Rehabilitación de las Construcciones

XVII International Conference on Pathology and Constructions Rehabilitation

FORTALEZA (Brasil), 3 a 5 de junho de 2021

https://doi.org/10.4322/CINPAR.2021.152

\title{
MAPA DE DANOS GERADO AUTOMATICAMENTE À PARTIR DA NUVEM DE PONTOS DE MÚLTIPLOS SENSORES PARA PATRIMÔNIO ARQUITETÔNICO - UMA REVISÃO SISTEMÁTICA DA LITERATURA
}

\section{Damage map automatically generated from the multiple sensor point cloud for architectural heritage - a literature systematic review}

\author{
RODRIGUES, Osvaldo Sélos ${ }^{1}$, KEMPTER, Eloisa Dezen² \\ ${ }^{1}$ UNICAMP, Limeira, Brasil, o035078@dac.unicamp.br \\ ${ }^{2}$ UNICAMP, Limeira, Brasil, elo@ft.unicamp.br
}

\begin{abstract}
Resumo: Os edifícios considerados de relevância histórica são cada dia mais numerosos nas cidades do Brasil e do mundo e os desafios para sua manutenção, preservação e restauro são complexos e necessitam de novas ferramentas tecnológicas para ganhar rapidez e eficiência em sua documentação. A plataforma HBIM (modelagem da informação da construção dedicada ao campo do patrimônio arquitetônico histórico) combinada com o levantamento digital do edifício realizado utilizando scaner à lazer terrestre e a fotogrametria de fotos captadas por drone são ferramentas inteligentes e precisas para alcançar o objetivo de documentar patrimônios arquitetônicos históricos com precisão e eficiência. Este artigo apresenta uma revisão sistemática da literatura publicada nessa temática nos últimos 5 anos (2015-2020) pois as tecnologias estão em exponencial desenvolvimento. Através da métodologia PRISMA com a utilização do programa StArt foram localizados 18 estudos que apresentam as ferramentas pré-definidas como eficientes e acessíveis para o levantamento digital preciso de patrimônio arquitetônico combinadas com a inteligência artificial para geração de mapa de danos. Este estudo conclui que as tecnologias na arquitetura e construção civil estão em alto ritmo de desenvolvimento com multiplos players trabalhando em diversas ferramentas, alguns possuem uma grande vantagem na interoperabilidade e é de grande ajuda na área da preservação de patrimônios encontrar as ferramentas mais eficientes e acessíveis para se estabelecer um protocolo para documentação inteligente dos edifícios históricos.
\end{abstract}

Palavras-chave: Patrimônio Arquitetônico, HBIM, Inteligência Artificial, Mapa de Danos.

Abstract: Buildings considered of historical relevance are increasingly numerous in the cities of Brazil and the world and the challenges for their maintenance, preservation and restoration are complex and need new technological tools to gain speed and efficiency in their documentation. The HBIM platform (building information modeling dedicated to the field of historical architectural heritage) combined with the digital survey of the building carried out using a terrestrial leisure scanner and photogrammetry of photos captured by drone are intelligent and accurate tools to achieve the goal of documenting heritage historical architecture with precision and efficiency. This article presents a systematic review of the literature published on this topic in the last 5 years (2015-2020) as the technologies are in exponential development. Through the PRISMA method using the StArt program, 18 studies were found that present the pre-defined tools as efficient and accessible for the accurate digital survey of architectural heritage combined with artificial intelligence to generate damage maps. This study concludes that technologies in architecture and civil construction are at a high pace of development with multiple players working on different tools, some have a great advantage 
in interoperability and it is of great help in the area of heritage preservation to find the most efficient and accessible tools to establish a protocol for intelligent documentation of historic buildings.

Keywords: Architectural Heritage, HBIM, Artificial Intelligence, Damage Map.

\section{Introdução}

Os edifícios considerados de relevância histórica são cada dia mais numerosos nas cidades do Brasil e do mundo e seus desafios para manutenção, preservação e restauro são complexos e necessitam de novas ferramentas tecnológicas para ganhar em rapidez e eficiência, visto que até hoje o levantamento da dados de edificações pré-existentes é realizado à mão com trena e papel, uma forma bastante rudimentar que não capta os ricos detalhes das obras e tão pouco as possíveis patologias presentes neles.

Uma ferramenta bastante eficiente, precisa e acessível atualmente para ajudar a documentar corretamente esses patrimônios históricos é o levantamento digital. Realizado por fotogrametria à partir de centenas, ou mesmo milhares, de fotos captadas por câmeras de alta precisão (algumas delas podendo inclusive estar à bordo de veículos aéreos não tripulados, os chamados drones ou vant, para captação das coberturas dos edifícios) ou por um scanner à laser que geram nuvens de pontos com cor e localização tridimensional, à partir da qual pode-se criar o gêmeo digital do edifício em programas que utilizam a plataforma BIM, onde o modelo digital tridimensional não é apenas parecido com o edifício original físico, mas seu banco de dados paramétricos e o enriquecimento semântico possível de se realizar nessa plataforma, o tornam realmente uma cópia mais fiel do prédio histórico, um documento para a posteridade, quando temos a consciência que os edifícios, por mais importantes e bem construído que sejam, não são eternos. Após esse processo podese utilizar a inteligência artificial para criar automaticamente mapas de danos de fachadas, documento este muito importante para os futuros trabalhos de restauro.

Françoise Choay (2006) conta em seu consagrado livro "A Alegoria do Patrimônio" que a primeira Comissão dos Monumentos Históricos foi criada em 1837 na França e objetivava a preservação dos edifícios remanescentes da Antiguidade, construções religiosas da Idade Média e alguns castelos. Com o tempo o interesse pela preservação arquitetônica cresceu e começou a abranger outros exemplares, incluindo arquitetura vernacular (construções populares sem a participação de arquitetos), conjunto de edifícios, bairros e até cidades inteiras ou conjunto de cidades conforme pode-se ver na lista do Patrimônio Mundial estabelecida pela Unesco.

O Brasil, assim como diversos outros países, precisa muito urgentemente de tecnologia, eficiência e precisão na documentação de seus patrimônios arquitetônicos históricos, ganhando assim agilidade e maior produtividade no setor de preservação devido à sua grande extensão territorial e baixa quantidade de proficionais habilitados para este serviço, pois a valorização dos patrimônios no Brasil é bastante recente e existem ainda poucos cursos nessa área.

Cogima (2019) relata que a preocupação com o patrimônio histórico só chegou ao Brasil por volta de 1920, e depois das primeiras discussões nacionais sobre o tema, e a criação do SPHAN (Serviço do Patrimônio Histórico e Artístico Nacional) em 1936, e que mais tarde (1970) viria a ser nomeado como IPHAN (Instituto do Patrimônio Histórico e Artístico Nacional), e que constitui o órgão máximo de preservação patrimonial na esfera nacional. Se compararmos o tempo do estudo da preservação no Brasil e na Europa podemos notar que estamos muito atrasados em relação aos europeus, porém mesmo com mais de 100 anos de estudos nacionais nessa área pouco ainda foi realizado.

Tolentino (2016) fala da preocupação com a precisão dos levantamentos e da demora em fazer os levantamentos mais precisos à mão, o que acaba deixando muitos patrimônios nacionais sem o devido registro, o que é um grande problema no caso de algum deles deixar de existir por algum motivo, o registro fiel pode vir a substituir parcialmente o prédio em questões da importância histórica, lembando dos recentes casos de incêndio no musue nacional do Rio de Janeiro e da Catedral de Notre Dame em Paris. O registro no tempo presente também é importante para a própria história do edifício, mostra como ele chegou após muitos anos até aqui, qual seu atual estado e toda essa documentação deve servir de base para a manutenção, preservação e restauro do patrimônio, como o mapa de danos, um instrumento de grande importância nesses processos. 
O objetivo desse estudo é fazer uma Revisão Sistemática da Literatura (RSL) publicada na área de preservação inteligente de patrimônio arquitetônico a fim de reconhecer as tecnologias mais indicadas e as metodologias mais eficientes disponíveis hoje em dia para a documentação inteligente. A RSL é de suma importância como base teórica de qualquer estudo pois evita que o pesquisador realize um estudo já existente, ajuda na busca por lacunas na bibliografia que possam gerar novos estudos relevantes e, se realizada com critérios bem definidos e explicitados, tornam o estudo mais confiável e replicável.

\section{Metodologia}

Para esta RSL foi escolhido o método PRISMA (Preferred Reporting Items for Systematic Reviews and MetaAnalyses) que consiste em um check list de tarefas que não podem ser omitidas em uma pesquisa científica, principalmente em artigos de review, e para este artigo o método foi utilizado para guiar o fluxograma de captação e análises de publicações relevantes ao tema abordado na pesquisa, portanto não foi seguido o check list à risca, e sim o fluxo do trabalho sugerido pelo método. Seguindo este método foi analisado o progresso das publicações sobre levantamento inteligente de dados de patrimônios históricos que utilizaram nuvem de pontos e inteligência artificial na literatura publicada em inglês nos últimos 5 anos (pois as tecnologias nessas áreas estão em constante desenvolvimento).

O primeiro passo da RSL é definir o string (expressão) de busca que será inserido nas bases de dados para selecionar de forma eficiente os trabalhos a serem analisados. A string definida para esta pesquisa foi dividida nas 3 principais etapas do projeto: Captura, Processamento e Obejtivo. E os termos foram utilizados em inglês, pois esta é a língua universal da pesquisa científica.

A Captura são os sensores que fazem o levantamento de dados atuais do edifício selecionado como estudo de caso, nesse caso foram utilizados 2 sensores para se obter uma nuvem de pontos completa e precisa, o escâner à laser terrestre e a câmera à bordo de um drone (ou vant - veículo aéreo não tripulado).

o Processamento é a parte em que os dados levantados do edifício pelos sensores são analisados e configurados no computador, o processo de fotogrametria com as fotos do drone, a nuvem de pontos gerada por cada sensor e combinadas e o ensino da inteligência artificial para identificação automática das patologias.

E o Objetivo é alcançar a documentação precisa e inteligente do patrimônio, para servir de base para sua manutenção e restauro: mapa de danos, identificação automática de patologias das fachadas e gêmeo digital em HBIM.

Figura 01 - String de Busca

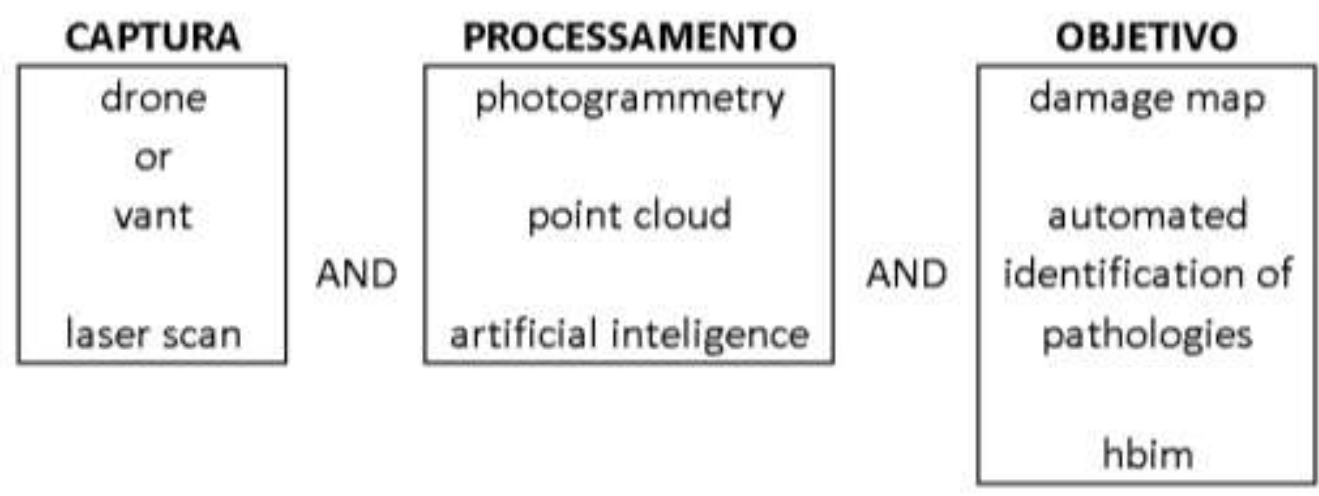

Fonte: Do autor

As principais bases de dados de publicações científicas com afinidade com a área do estudo foram identificadas: Web of Science, Springer, Science Direct, Scopus, Scielo, ACM, IEEE Xplore e ASCE. 
Em Web of Science e Springer foram identificadas dificuldades de utilizar a string definida, o que fez elas serem descartadas da RSL. A ASCE não encontrou resultados para a busca.

Em Scopus e IEEE Xplore os resultados encontrados não puderam ser exportados como arquivo BBTEX, o que se faz necessário para utilização do programa que foi escolhido para as análises dos dados, o StArt (State of the Art Throug Systematic Review), o que fez elas também serem descartadas.

As bases utilizadas portanto foram Science Direct e ACM.

Para extração e análises dos resultados das pesquisas nas bases de dados foi utilizado o programa StArt (State of the Art Throug Systematic Review), um software desenvolvido pelo LaPES UFSCAR e distribuído gratuitamente pelo site da universidade que segue o padrão da metodologia PRISMA. Este programa open source foi de grande utilidade no desenvolvimento desta RSL.

\section{Figura 02 - Fluxo da RSL}
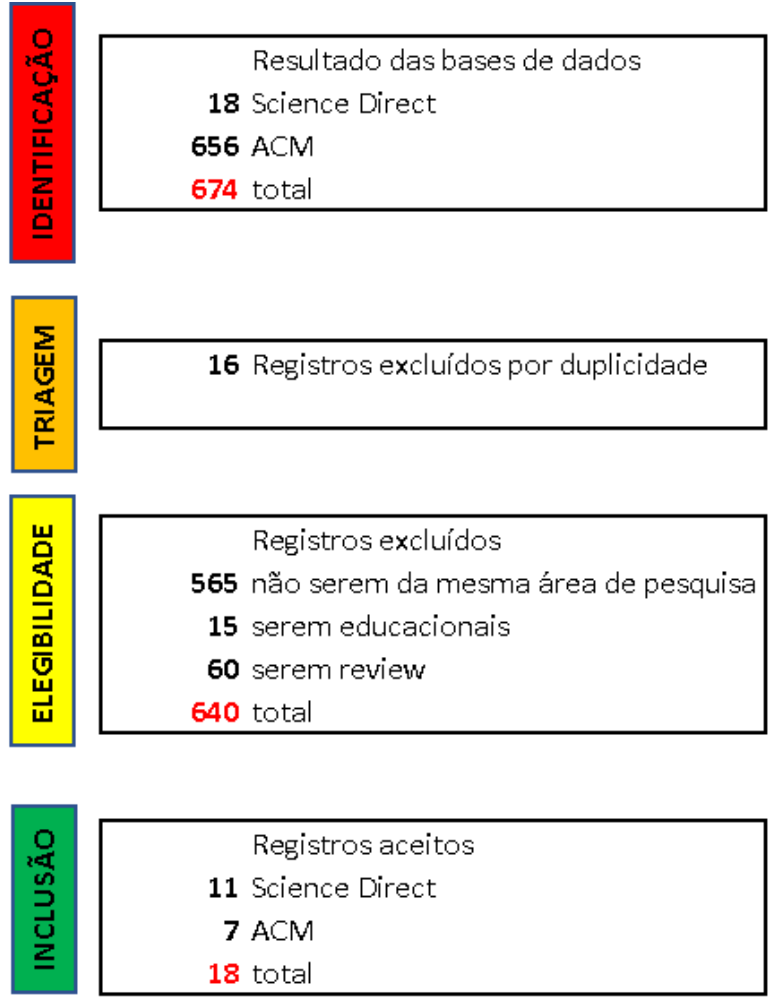

Fonte: Do autor

Gráfico 01 - Gráfico do balanço de resultados da RSL

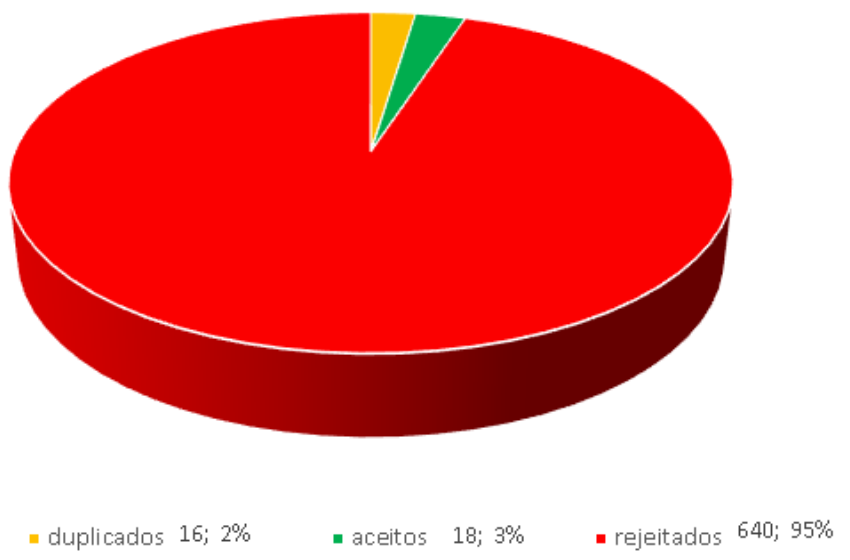

Fonte: Do autor 


\section{Resultados}

No gráfico 02 foram demonstrados os resultados de quantidades de artigos identificados separados pelas bases utilizadas (em azul), observa-se também a quantidade desses artigos que foram rejeitados (em vermelho) e aceitos (em verde) e a taxa de aceitação por base de dados (linha laranja). Pode-se observar que a base de dados que obteve maior número de resultados identificados (656) pela string utilizada foi a ACM, porém ela também teve um alto índice de reprovação de artigos (649), onde somente $0,01 \%$ deles foram aceitos para a RSL (7), uma possível explicação para este fenômeno pode ser que a base de dados não tenha trabalhado os operadores boleanos de forma correta, mostrando assim trabalhos científicos que apresentavam somente palavras de um grupo de trabalho, o que abriria muito o leque de áreas do conhecimento afetadas.

Gráfico 02 - Porcentagem dos resultados por base de dados

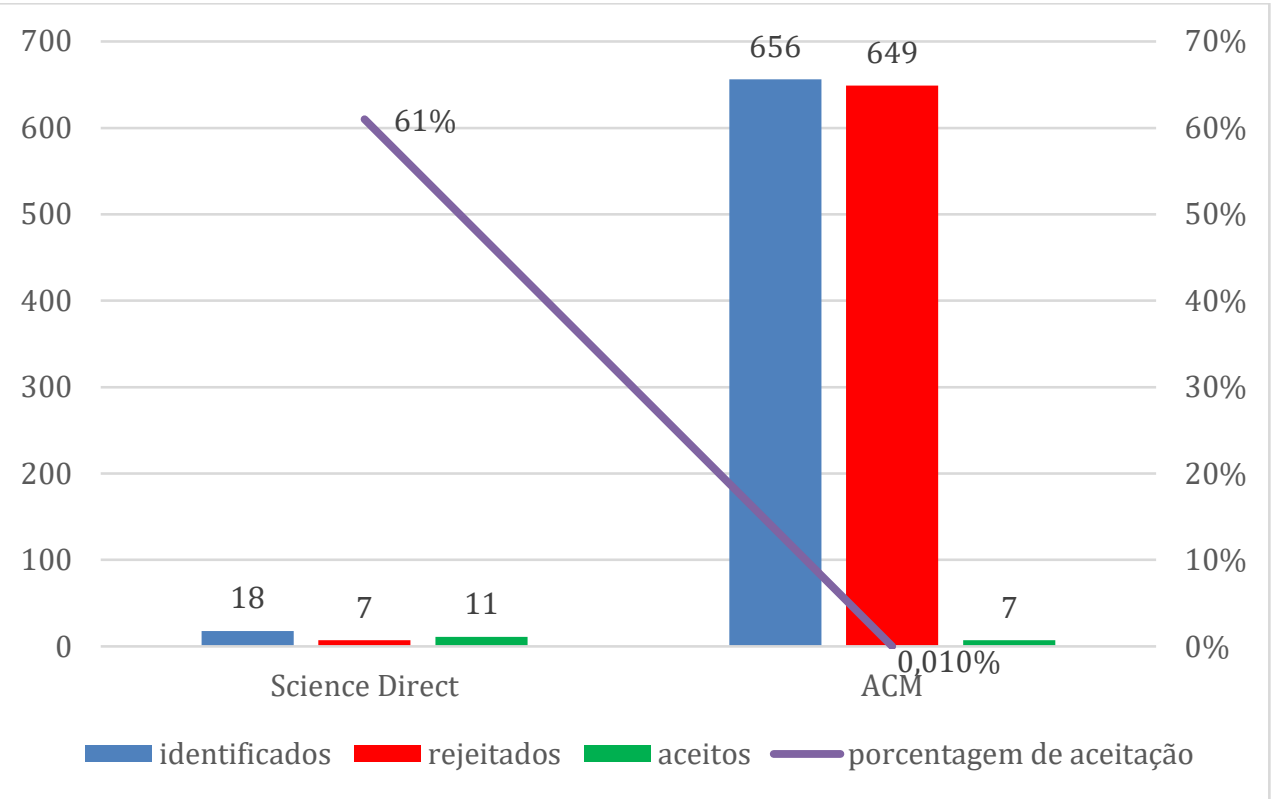

Fonte: Do autor

Na figura 04 pode-se observar um mapa de ocorrência de palavras chave dos artigos aceitos na RSL, notase em destaque maior as palavras que mais apareceram como Data (dados), Cloud (nuvem), Al (inteligência artificial) entre outras. Com esse resultado firma-se que a string de busca foi determinante na identificação dos trabalhos nas bases de dados pesquisadas.

Figura 03 - Frequência das palavras-chave nos artigos aceitos

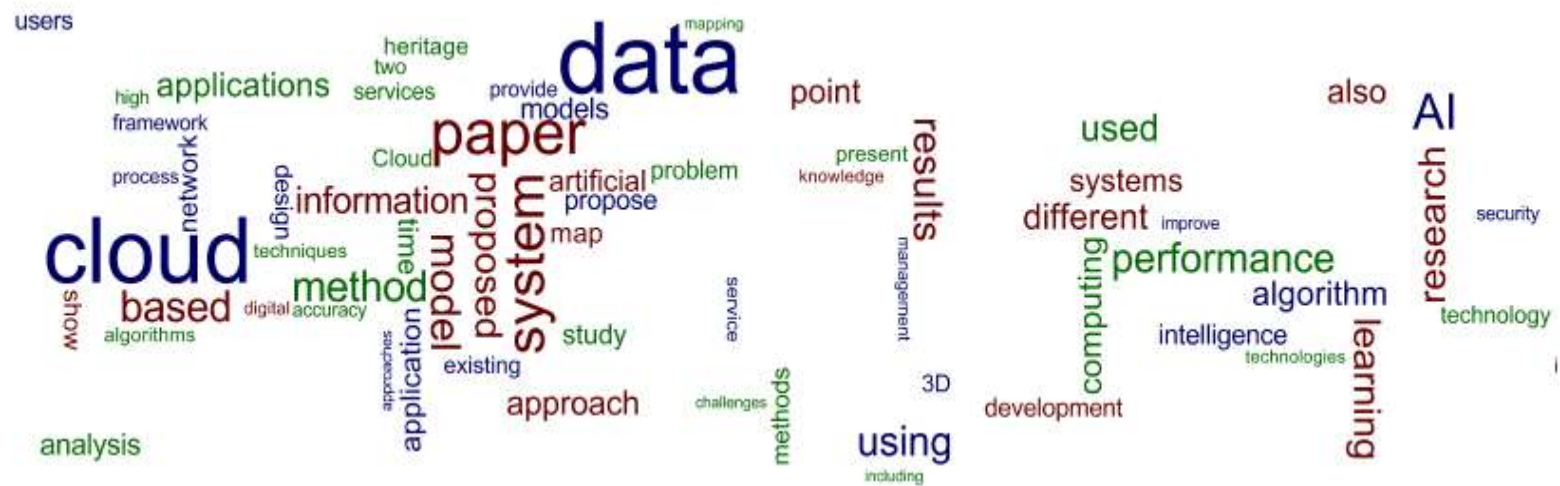

Fonte: Do autor 
No gráfico 03 demonstra-se os anos de publicações dos artigos aceitos na RSL, onde pode-se observar uma forte tendência de crescimento de pesquisas nessa área nos últimos 5 anos (essas buscas foram realizadas em janeiro de 2021 e restritas nas publicações entre 2015-2020).

Gráfico 03 - Ano de publicação dos artigos aceitos

\section{quantidade de publicações aceitas por ano}

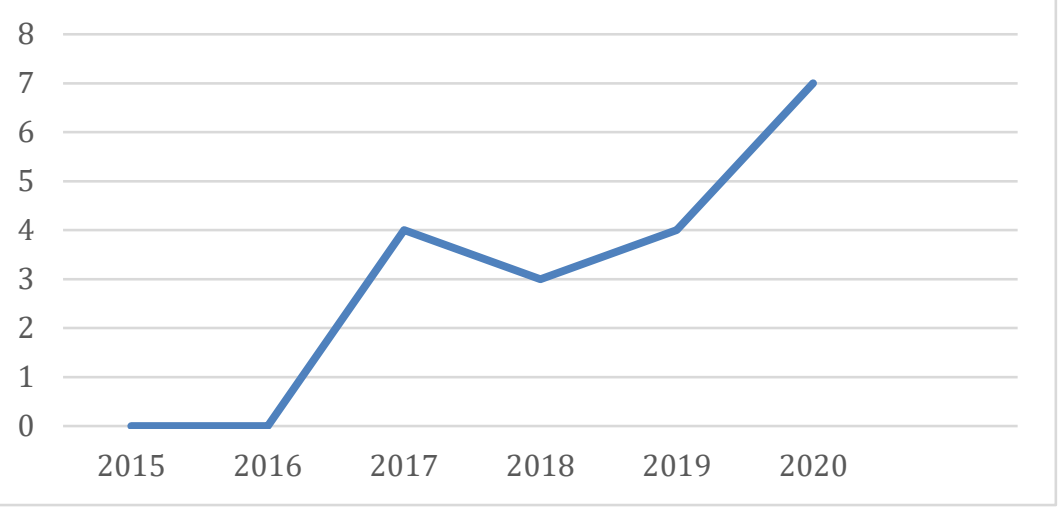

Fonte: Do autor

\section{Discussão}

Neste quadro do estado da arte do campo de pesquisa deste trabalho é demonstrado um grande aumento do interesse nas pesquisas relacionadas à patrimônio cultural e levantamentos tecnológicos eficientes de edificações históricas nos últimos anos, o que é acompanhado do avanço das tecnologias que podem influenciar essa atividade, como drones com sistemas de voos mais precisos e câmeras mais potentes, escâneres à laser mais acessíveis e mais precisos e diversos novos softwares que automatizam de forma eficiente o trabalho de processamentos de dados complexos como grandes nuvens de pontos. No quadro 01 observa-se os títulos originais dos 18 artigos selecionados na RSL com seus respectivos autores, ano e meio de publicação.

Quadro 01 - Identificação dos artigos selecionados na RSL

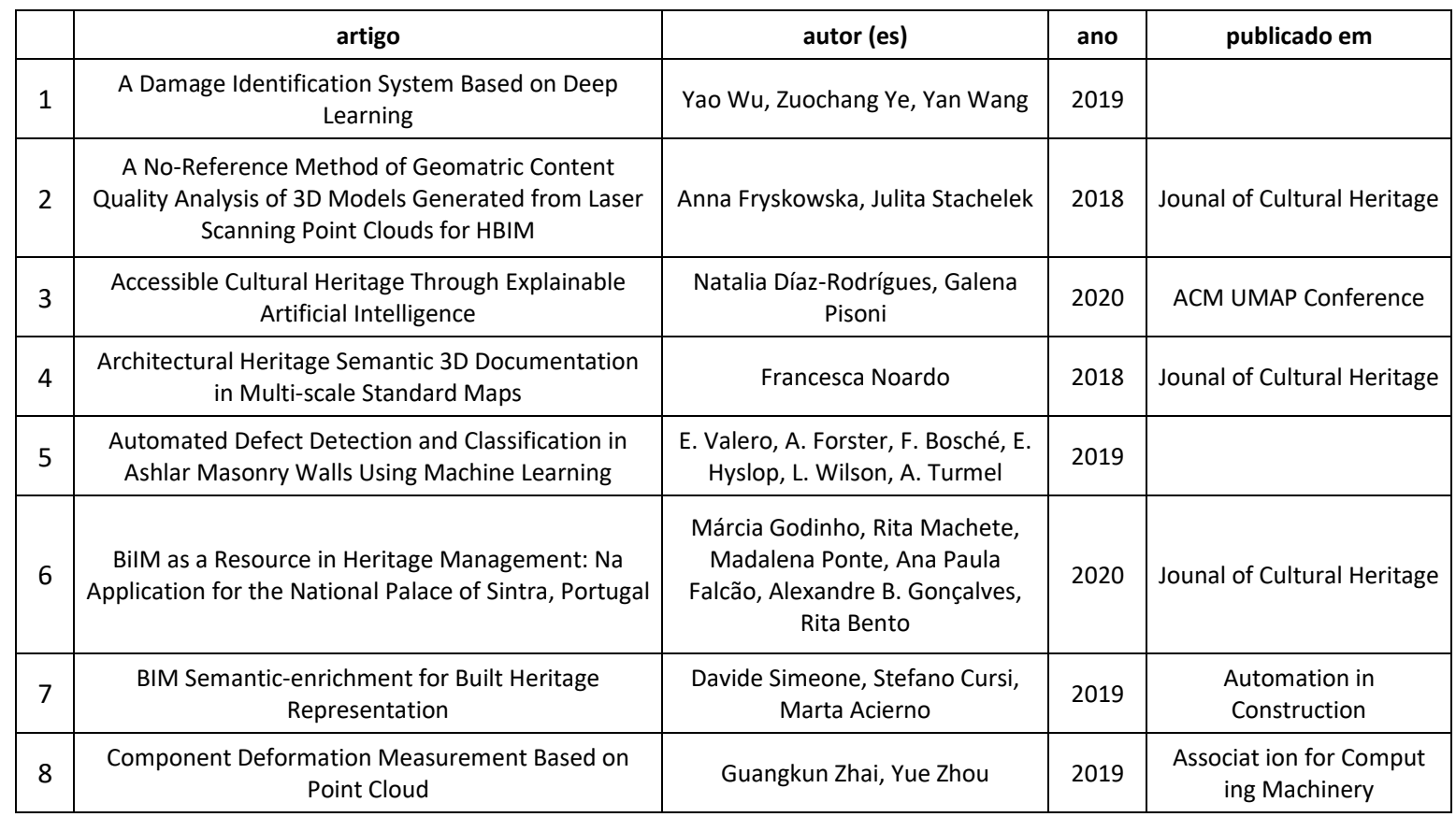




\begin{tabular}{|c|c|c|c|c|}
\hline 9 & $\begin{array}{l}\text { Digital Anastylosis of the Remains of a Portal by } \\
\text { Master Builder Hérnan Ruiz: Knowledge Strategies, } \\
\text { Methods and Modelling Results }\end{array}$ & $\begin{array}{l}\text { Roque Ângulo, Francisco Pinto, } \\
\text { Jesús Rodrígues, Antonio } \\
\text { Palomino }\end{array}$ & 2017 & $\begin{array}{l}\text { Digital Applications in } \\
\text { Archaeology and Cultural } \\
\text { Heritage }\end{array}$ \\
\hline 10 & $\begin{array}{l}\text { From Point Cloud to Jeddah Heritage BIM Nasif } \\
\text { Historical House - Case Study }\end{array}$ & Ahmad Baik & 2017 & $\begin{array}{c}\text { Digital Applications in } \\
\text { Archaeology and Cultural } \\
\text { Heritage }\end{array}$ \\
\hline 11 & $\begin{array}{l}\text { Historic Building Information Modelling: } \\
\text { Performance Assessment for Diagnosis-aided } \\
\text { Information Modelling and Management }\end{array}$ & $\begin{array}{l}\text { Silvana Bruno, Mariella de Fino, } \\
\text { Fabio Fatiguso }\end{array}$ & 2018 & $\begin{array}{l}\text { Automation in } \\
\text { Construction }\end{array}$ \\
\hline 12 & $\begin{array}{c}\text { Knowled-based Data Enrichment for HBIM: Exploring } \\
\text { High-quality Models Using the Semantic-web }\end{array}$ & $\begin{array}{l}\text { Ramona Quattrini, Roberto } \\
\text { Pierdicca, Christian Morbidoni }\end{array}$ & 2017 & $\begin{array}{l}\text { Journal of Cultural } \\
\text { Heritage }\end{array}$ \\
\hline 13 & $\begin{array}{l}\text { Learning From Synthetic Point Cloud Data for } \\
\text { Historical Buildings Semantic Segmentation }\end{array}$ & $\begin{array}{c}\text { Christian Morbidoni, Roberto } \\
\text { Pierdicca, Marina Paolanti, } \\
\text { Ramona Quattrini, Raissa } \\
\text { Mammoli }\end{array}$ & 2020 & $\begin{array}{l}\text { ACM Journal on } \\
\text { Computing and Cultural } \\
\text { Heritage }\end{array}$ \\
\hline 14 & $\begin{array}{l}\text { Literature Review of Building Information Modeling } \\
\text { (BIM) Intended for the Purpose of Renavation } \\
\text { Projects }\end{array}$ & $\begin{array}{l}\text { Laurent Joblot, Thomas Paviot, } \\
\text { Dominique Deneux, Samir } \\
\text { Lamouri }\end{array}$ & 2017 & \\
\hline 15 & $\begin{array}{c}\text { On the Accuracy of UAV Photogrammetry Survey for } \\
\text { the Evaluation of Historic Masonry Strutural } \\
\text { Damages }\end{array}$ & $\begin{array}{c}\text { Nicola Cavalagli, Massimiliano } \\
\text { Gioffrè, Silvia Grassi, Vittorio } \\
\text { Gusella, Chiara Pepi, Gian Marco } \\
\text { Volpi }\end{array}$ & 2020 & \\
\hline 16 & $\begin{array}{l}\text { The Effect of VR Environments on the Acceptance, } \\
\text { Experience and Expectations of Cultural Heritage } \\
\text { Learning }\end{array}$ & $\begin{array}{l}\text { Eugene Ch'ng, Yue Li, Shengdan } \\
\text { Cai, Fui-theng Leow }\end{array}$ & 2020 & $\begin{array}{l}\text { ACM Journal on } \\
\text { Computing and Cultural } \\
\text { Heritage }\end{array}$ \\
\hline 17 & $\begin{array}{c}\text { Towards de Semantic-Aware 3D Digitisation of } \\
\text { Architectural Heritage: The "Notre-Dame de Paris" } \\
\text { Digital Twin Project }\end{array}$ & Livio de Luca & 2020 & SUMAC20 \\
\hline 18 & $\begin{array}{l}\text { Weakly Supervised 3D Object Detection from Point } \\
\text { Clouds }\end{array}$ & Zengyi Qin, Jinglu Wang, Yan Lu & 2020 & \\
\hline
\end{tabular}

Fonte: Do autor

Entre as principais conclusões dos artigos lidos encontram-se: falhas de interoperabilidade de softwares necessários para o processamento de dados dos levantamentos e como esse quesito é importante para todos os processos e etapas da documentação inteligente de patrimônios, comparações entre as diferentes tecnologias encontradas para essa finalidade atualmente, quanto aos seus custos, velocidade, eficiência e precisão, as vantagens da combinação de nuvens de pontos de multiplos sensores complementares, os mais citados são fotogrametria e varedura à laser, as grandes facilidades para manutenção dos edifícios históricos com a utilização da plataforma BIM, a importância de se estabelecer um metodologia base para os levantamentos serem mais eficientes, as necessidades urgentes dos levantamento digitais chegarem ao campo de trabalho efetivo da preservação e manutenção de patrimônios arquitetônicos pois as técnicas atuais manuais não são capases de realizar todo o trabalho existente e de forma satisfatória e como vem crescendo a utilização de inteligencia artifical em diversos campos e que ela pode ser um grande avanço para esses processos de levantamentos digitais.

\section{Conclusões}

O presente estudo teve como objetivo analisar o panorama atual das principais pesquisas dos últimos 5 anos em HBIM e documentação inteligente de patrimônios arquitetônicos. Para tanto foi realizada uma RSL que resultou em 18 artigos para análise completa.

Pelas análises realizadas nesse artigo é possível afirmar que o interesse em preservação de patrimônios arquitetônicos vem crescendo muito no Brasil e no mundo todo e que esse aumento no interesse da área implica em conseguir documentar e preservar melhor e mais rápido esses edifícios sensíveis ao tempo, às intempéries e à ação humana. A falta de profissionais capacitados e recursos financeiros impulsiona as 
pesquisas em descobertas, desenvolvimentos e metodologias tecnológicas eficientes, precisas e acessíveis para realização de levantamentos digitais dos patrimônios.

Por fim identificamos que as tecnologias e metodologias para documentação inteligente e precisa de patrimônios mais eficientes e acessíveis segundos os estudos analisados foram: levantamento digital do patrimônio através de nuvens de pontos combinadas de dois diferentes sensores, o scanner à laser terrestre e a fotogrametria com fotos aéreas captadas por câmeras de precisão abordo de drones, o digital twin realizado a partir dessa nuvem de pontos na plataforma BIM e a utilização da inteligência artifical para geração automatizada de um mapa de danos das fachadas do edifício.

\section{Agradecimentos}

O presente trabalho foi realizado com apoio da Coordenação de Aperfeiçoamento Pessoal de Nível Superior

- Brasil - (CAPES) - Código de Financiamento 001.

\section{Referências Bibliográficas}

Ângulo, R., Pinto, F., Rodrígues, J., Palomino, A. (2017). Digital Anastylosis of the Remains of a Portal by Master Builder Hérnan Ruiz: Knowledge Strategies, Methods and Modelling Results. University of Seville, Spain.

Baik, A. (2017). From Point Cloud to Jeddah Heritage BIM Nasif Historical House - Case Study. University College London, UK.

Bruno, S., Fino, M., Fatiguso, F. (2018). Historic Building Information Modelling: Performance Assessment for Diagnosis-aided Information Modelling and Management. Polytechnic of Bari, Italy.

Cavalagli, N., Gioffrè, M., Grassi, S., Gusella, V., Pepi C., Volpi, G.M. (2020). On the Accuracy of UAV Photogrammetry Survey for the Evaluation of Historic Masonry Strutural Damages. University of Perugia, Italy.

Choay, F.; A Alegoria do Patrimônio; 5̣ edição, São Paulo: Estação Liberdade: UNESP, 2006.

Cogima, C. K.; Bim Aplicado ao Patrimônio Histórico Baseado em Levantamento Híbrido com Multisensores; UNICAMP, Limeira, 2019.

Ch'ng, E., Li, Y., Cai, S.,Leow, F.T. (2020). The Effects of VR Environments on the Acceptance, Experience, and Expectations of Cultural Heritage Learning. ACM J. Comput. Cult. Herit. 13, 1, Article 7 (February 2020).

Fryskowska, A., Stachelek, J. (2018). A No-Reference Method of Geomatric Content Quality Analysis of 3D Models Generated from Laser Scanning Point Clouds for HBIM. Military University of Technology, Warsaw, Poland.

Godinho, M., Machete, R., Ponte, M., Falcão, A.P., Gonçalves, A.B., Bento, R. (2020). BilM as a Resource in Heritage Management: Na Application for the National Palace of Sintra, Portugal. Universidade de Lisboa, Portugal.

Joblot, L., Paviot, T., Deneux, D., Lamouri, S. (2017). Literature Review of Building Information Modeling (BIM) Intended for the Purpose of Renavation Projects. Arts et Métiers Paris Tech, Paris, France.

Luca, L. (2020). Towards the Semantic-aware 3D Digitisation of Architectural Heritage: The "Notre-Dame de Paris" Digital Twin Project. SUMAC'20, October 12, 2020, Seatle, WA, USA.

Morbidoni, C., Pierdicca, R., Paolanti, M., Quattrini, R., Mammoli, R. (2020). Learning From Synthetic Point Cloud Data for Historical Buildings Semantic Segmentation. Universitá Politecnica dele Marche, Italy.

Noardo, F. (2018). Architectural Heritage Semantic 3D Documentation in Multi-scale Standard Maps. Politecnico di Torino, Italy.

Qin, Z., Wang, J., Lu, Y. (2020). Weakly Supervised 3D Object Detection from Point Clouds. In M '20: ACM Multimedia, October 12-16, 2020, Seattle, United States. ACM, New York, NY, USA.

Quattrini, R., Pierdicca, R., Morbidoni, C. (2017). Knowled-based Data Enrichment for HBIM: Exploring Highquality Models Using the Semantic-web. Universitá Politecnica dele Marche, Italy.

Rodrígues, N.D., Pisoni, G. (2020). Accessible Cultural Heritage Through Explainable Artificial Intelligence. Institut Polytechnique Paris, France. 
Simeone, D., Cursi, S., Acierno, M. (2019). BIM Semantic-enrichment for Built Heritage Representation. Sapienza University of Rome, Italy.

Tolentino, M. M. A.; A Utilização do HBIM na Documentação, na Gestão e na Preservação do Patrimônio Arquitetônico; SIGraDi 2016, XX Congresso f the Iberoamerican Society of Digital Graphics, Buenos Aires, Argentina, 2016.

Valero, E., Forster, A., Bosché, F., Hyslop, E., Wilson, L., Turmel, A. (2019). Automated Defect Detection and Classification in Ashlar Masonry Walls Using Machine Learning. The University of Edinburgh, UK.

Wu, Y., Ye, Z., Wang, Y. (2019). A Damage Identification System Based on Deep Learning. ICIT, Shanghai, China.

Zhai, G., Zhou, Y. (2019). Component Deformation Measurement Based on Point Cloud. ICIT, Shanghai, China. 